\title{
EUV Focus Sensor: Design and Modeling
}

\author{
Kenneth A. Goldberg ${ }^{*}$, , Maureen E. Teyssier ${ }^{2}$, J. Alexander Liddle ${ }^{1}$ \\ ${ }^{1}$ Center for X-Ray Optics, Lawrence Berkeley National Laboratory, Berkeley, CA 94720 \\ ${ }^{2}$ Department of Physics, Univ. of California, Berkeley, Berkeley, CA 94720
}

\begin{abstract}
We describe performance modeling and design optimization of a prototype EUV focus sensor (FS) designed for use with existing 0.3-NA EUV projection-lithography tools. At 0.3-NA and 13.5-nm wavelength, the depth of focus shrinks to 150 $\mathrm{nm}$ increasing the importance of high-sensitivity focal-plane detection tools. The FS is a free-standing Ni grating structure that works in concert with a simple mask pattern of regular lines and spaces at constant pitch. The FS pitch matches that of the image-plane aerial-image intensity: it transmits the light with high efficiency when the grating is aligned with the aerial image laterally and longitudinally. Using a single-element photodetector, to detect the transmitted flux, the FS is scanned laterally and longitudinally so the plane of peak aerial-image contrast can be found.

The design under consideration has a fixed image-plane pitch of 80-nm, with aperture widths of 12-40-nm (1-3 wavelengths), and aspect ratios of 2-8. TEMPEST-3D is used to model the light transmission. Careful attention is paid to the annular, partially coherent, unpolarized illumination and to the annular pupil of the Micro-Exposure Tool (MET) optics for which the FS is designed. The system design balances the opposing needs of high sensitivity and high throughput optimizing the signal-to-noise ratio in the measured intensity contrast.
\end{abstract}

Keywords: extreme ultraviolet lithography, focus sensor, aerial image monitor.

\section{INTRODUCTION}

A new generation of $5 \times$ demagnification, 0.3 numerical aperture (NA), prototype extreme ultraviolet (EUV) optical systems is being produced to provide opportunities for early learning with sub-50-nm EUV imaging. Known as MicroExposure Tools ${ }^{1}$ (MET), these systems have been developed for static micro-field imaging, featuring diffraction-limited projection optics for 13.5-nm-wavelength illumination.

At 13.5 -nm wavelength and $0.3 \mathrm{NA}$, the depth of focus shrinks to approximately $150-\mathrm{nm}$. In principle, a high-sensitivity focus sensor (FS) installed on the wafer stage can simplify the process of tracking focus in a tool. A FS tool probes the aerial image formed in the wafer plane by appropriately designed mask features; it thus offers a distinct advantage over position sensors that reference the external housing of the projection lens and do not sample the image directly. Furthermore, if configured in multiple directions, such a tool could be used to measure astigmatism in the projection lens.

Here we describe the design optimization of a relatively simple FS tool. A mask pattern of constant-pitch lines and spaces is illuminated in a standard way by the system's EUV light source. The light source has an annular angular spectrum with $\sigma$ values between 0.5 and 0.7. ( $\sigma=1$ corresponds to the largest acceptance angle of the entrance pupil, which is 0.06 NA.) The FS measures the aerial image in the wafer-plane where the mask-grating's image is formed. A freestanding Ni grating of constant pitch, aligned to the direction of the mask lines, is scanned in the lateral and longitudinal directions. Where the open regions of the grating align with the bright (high intensity) regions in the aerial image, the transmission is high. The FS can be shifted by a half-pitch distance to reduce the transmission to its minimum value. Out of the focal plane in either direction, the measured contrast between maximum and minimum throughput decreases and in this way, the focal plane can be identified.

With a fixed grating pitch of 80-nm, fixed annular illumination as described above, and a simple 1:1 line-space mask pattern (400-nm-pitch in the mask plane), the primary design parameters under consideration in this study are the Ni

*KAGoldberg@lbl.gov; phone 1510 495-2261; fax 1510 486-4550; www-cxro.lbl.gov 
grating thickness and its "open width" defined as the uniform free-space width of the line openings in the grating. For the purposes of this study, we restrict the parameter space to the following ranges. Thicknesses between 80 and $100 \mathrm{~nm}$ are considered. Thicker gratings reduce the throughput and make the fabrication of high-aspect-ratio structures more difficult without offering significant advantages; thinner gratings provide lower absorption in the anti-aligned position, and may be extremely fragile. Open-width values range from 12 to $40 \mathrm{~nm}$. A grating with narrow openings may offer higher field measurement fidelity at the significant expense of flux.

The design optimization is considered from the standpoint of signal measurement: an optimized design is one that maximizes the signal-to-noise ratio in the contrast measurement. While the practical limitations of fabrication and use may dictate that other factors be included in the design optimization, the model presented here provides a good starting point and an analytical framework for the system.

\section{SIMULATION METHOD}

Simulating the performance of the FS entails several steps: an overview is given here, and the individual steps are described in greater detail below. The coherence properties of the light source are included through the partially coherent addition of plane-wave solutions, following the Hopkins formulation ${ }^{2,3}$. The distributed source is discretized, and complete field calculations are made separately from individual, coherent, plane-wave sources. We treat the mask reflection as an ideal, reflective, 1:1 line:space, square grating. (Mask-dependent refinements could be included in future studies to accommodate second-order effects.) The mask diffraction pattern, which includes the off-axis illumination, is projected into the MET's annular pupil, and the system's transmission is calculated on a discrete grid. At this point, the positions of non-zero intensity in the exit pupil are treated as mutually coherent plane-wave "source" points for the aerial image formation. In the FS transmission calculations, the aerial image is never calculated directly. (However, for comparison with a thin opaque screen analog, the aerial image is calculated.) TEMPEST-3D ${ }^{4,5}$ (described in Section 2.1) is used to model the transmission of plane waves through the FS grating; the specific plane-wave angles used in the TEMPEST-3D calculations match the grid used in the representation of the pupil transmission. Hence the complex field amplitudes in the exit pupil (coming from the grating diffraction of a single source point) are the plane wave coefficients used in a coherent addition.

\subsection{Aerial Image}

The aerial image through focus defines the local field environment of the FS; understanding the aerial image enhances our understanding of the tool's performance. While coherent illumination would create a grating pattern with a very large focal depth, the partially coherent illumination used in this study concentrates the region of high contrast to a narrow $z$ range close to focus. Outside of this narrow range, the partially coherent nature of the aerial image forms regions of low field contrast. The annular pupil and annular illumination profile $(0.5<\sigma<0.7)$ strongly influence the aerial image. The field's polarization also influences the aerial image, to a lesser degree: in these calculations orthogonal polarizations are combined in intensity to generate the unpolarized solution.

Figure 1 contains a cross-sectional view of two spatial periods of the field. The plots to the right show the minimum and maximum field intensity values in each $z$ (longitudinal) plane. From these values, the inherent field contrast is calculated according to the formula: contrast $=(\max -\min ) /(\max +\min )$. From a peak value of 0.688 the field contrast decreases symmetrically away from the focal plane, with a full-width at half-maximum (FWHM) of $361 \mathrm{~nm}$, and a minimum value of 0.042 at approximately $500 \mathrm{~nm}$ from focus. The longitudinal depth of the inherent field contrast is important to the sensitivity of the FS tool since the measured contrast will be used to assess the location of the focal plane.

\subsection{TEMPEST-3D Simulations}

TEMPEST-3D calculations are the central element of the FS modeling. TEMPEST-3D is a time-domain vector electromagnetic field simulation tool that calculates the interaction of light and matter. Using an arbitrary input field source, and a discrete simulation domain in which the index of refraction is defined in each volume element, TEMPEST-3D solves Maxwell's Equations at each point in space, iterating until a self-consistent, steady state solution with arbitrary, given precision is found. 

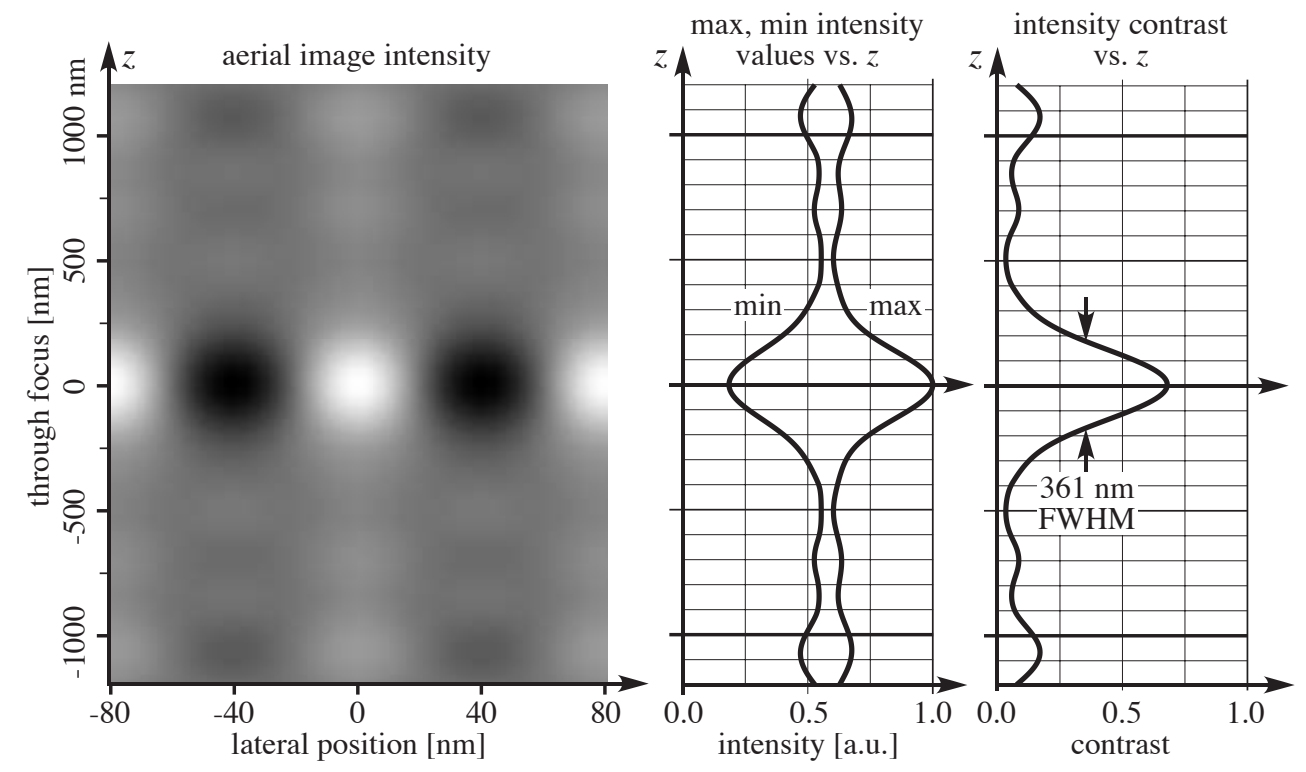

Fig 1. Calculated partially-coherent throughfocus aerial image for unpolarized light. Two periods of the pattern are shown in the intensity cross-section image at left. At right (with vertical axes matching that of the cross-section) are the extracted field intensity minimum and maximum values in each $z$ plane, and the inherent field contrast calculated from those values.

The FS simulation takes advantage of translation invariance in the direction of the grating lines to reduce the simulation to two dimensions: the $x z$ plane, perpendicular to the grating lines. Periodic boundary conditions are imposed at the lateral boundaries to create an infinite virtual grating. The discrete simulation domain includes $12.67 \mathrm{~nm}$ above and $5.33 \mathrm{~nm}$ below the grating structure with free-space surrounding the Ni absorber regions. The grid spacing used the FS simulation is $0.67 \mathrm{~nm}$ per node. Within TEMPEST-3D, the simulation domain is also periodic in the propagation, $z$-direction. To block the transmitted light from coming back through the top of the domain, the top and bottom areas of the domain are lined with nonphysical perfectly-matched layers ${ }^{6}$ which, for a finitedifference time-domain (FDTD) model like TEMPEST3D, are designed to absorb all incident light.

The source plane, located $12.67 \mathrm{~nm}$ above the top of the Ni grating, introduces tilted plane-waves into the domain. Since the source electric field must follow the periodic boundary condition, only a discrete set of angles are allowed: the electric-field phase values must be continuous across the lateral boundaries into the adjacent virtual domains. This condition is satisfied for a domain width, $w$, by allowed angles, $\theta_{n}$, with integer $n$, following Eq. (1).

$$
\sin \theta_{n}=n \lambda / w .
$$

Increasing the lateral simulation domain width (i.e. including more periods of the grating) allows a greater number (and density) of illumination angles to be included. We chose to set $w$ equal to 10 cycles of the grating, or $800 \mathrm{~nm}$. Within an NA of 0.3 (the sine of the largest angle in the exit pupil is $0.3 ; \sin \theta_{\max }=0.3$ ), this domain width enabled a total of 35 orders to be used in the simulation, $-17 \leq n \leq 17$.

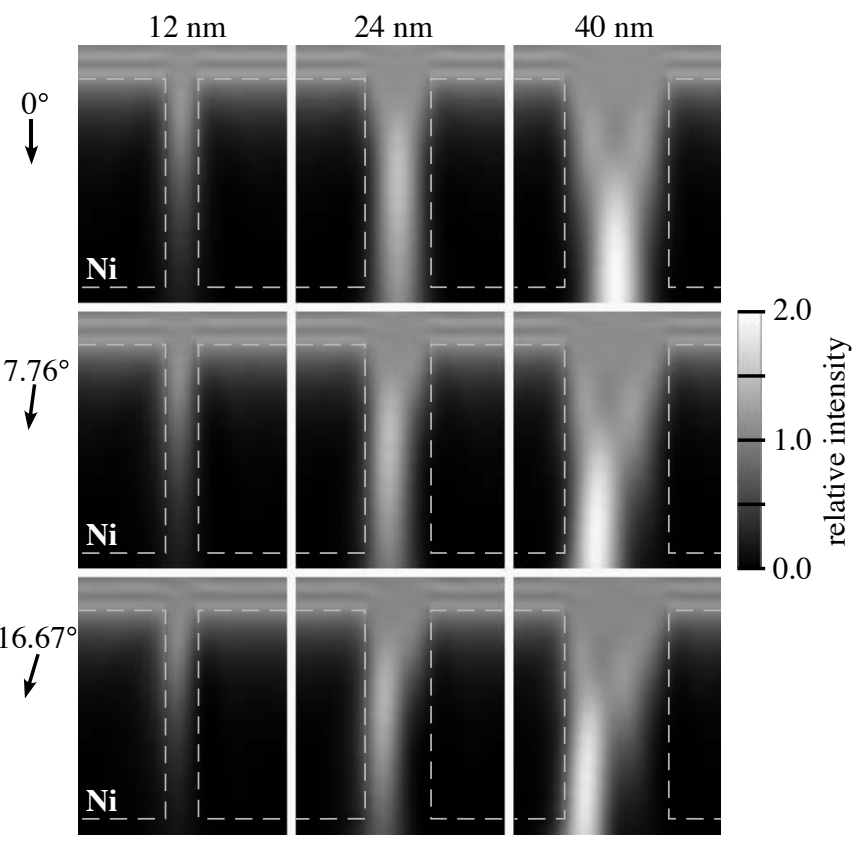

Fig 2. Field intensity cross sections for unpolarized plane-wave illumination, calculated with TEMPEST-3D. The incident field has unit intensity. The boundaries of the 80-nm-thick Ni grating are shown by the dashed lines. Three different grating open-widths and angles of incidence are shown. Individual calculations such as these are combined to form the partially coherent transmitted field. 
Nine examples of individual TEMPEST-3D calculations are shown in Fig. 2. Field intensity cross-sections are plotted for three different grating open-widths $(12,24$, and $40 \mathrm{~nm})$ and three incident angles.

\subsection{Light Propagation and Partially Coherent Addition}

Modeling the partially coherent illumination, requires that the source be discretized and treated as a set of individual source points. For each discrete source point, the calculation of mask-reflection, lens-transmission, and FS-grating transmission is handled in a fully-coherent manner, wherein the field amplitudes and phases are preserved. When the fields from the individual source points are combined, they are treated as mutually incoherent and they are added together by intensity. Furthermore, calculations are performed separately for $x$ and $y$ polarizations, and their intensities are combined to simulate unpolarized illumination.

In this simulation, the source follows an annular angular illumination pattern spanning $\sigma$ values between 0.5 and 0.7 . The discretization renders the source onto a $19 \times 19$ grid domain that approximates the continuous source, using a continuous range of intensity values from 0 to 1 at each point.

Neglecting second order mask-reflection effects arising form the surface topography and off-axis illumination, we model the reflection from the binary opaque-and-transparent mask as a series of diffraction orders of ideal strengths and sharply defined diffraction angles. Each source point under consideration has a given angle of incidence in the $x$ and $y$ direction; this angle is preserved as the diffraction orders are propagated into the pupil. The annular angular acceptance of the MET pupil (30\% central obscuration) is applied to the transmission of the diffracted light. Since the TEMPEST-3D simulations are performed only for discrete incident angles, the light distribution in the pupil (defined with continuous angles) must be binned (i.e. added into discrete groups by angle) to match. At this point, the polarization of the light is taken into account so that the recombination in the image-plane of light from different angles and polarizations will be physically correct.

\section{DESIGN OPTIMIZATION}

The primary metrics of the FS performance are the transmission efficiency in the (laterally) aligned and anti-aligned positions, and the measurement contrast calculated from the transmission values. Since the contrast is used to establish the image-plane position, the optimization process seeks the design with the highest signal-to-noise ratio (SNR) in the contrast measurement for a given illumination flux level. The SNR will always increase with higher flux.

Operation of the FS requires repeated measurements of the local field contrast, performed by reading the maximum and minimum transmittance values as the FS is translated laterally. Within the periodic aerial image, the extrema should be found one half-cycle apart, $40 \mathrm{~nm}$. Optimizing the signal-to-noise ratio in the contrast measurements can be performed as follows.

Consider the incident photon flux on the grating structure to be $k$ photons per measurement time. The maximum flux, $A$, transmitted through a given grating design is defined as $k a$, where $a$ is a dimensionless function of the design parameters, the illumination conditions, and the longitudinal FS position. The function $a$ ranges from 0 and 1 . Similarly, the minimum transmitted flux, $B$, is $k b$, and $b$ is also a function of the design. $b$ ranges from 0 to $a$.

The measurement contrast, $C$, is a function of $A$ and $B$ and therefore also depends on all design and illumination parameters. Since $C$ describes the way light passes through the FS structure, it is not the same as the field contrast. $C$ is defined as

$$
C=\frac{A-B}{A+B} \text {. }
$$

The standard deviation of the contrast measurement, $\sigma_{C}$, depends on the variances of $A$ and $B$. Following Poisson statistics for noise in photon counting, the uncertainties in the independently measured quantities, $A$ and $B$, are, respectively,

$$
\begin{gathered}
\sigma_{A}=\sqrt{A}, \text { and } \sigma_{B}=\sqrt{B} \\
\sigma_{C}^{2}=\left(\frac{\partial C}{\partial A} \sigma_{A}\right)^{2}+\left(\frac{\partial C}{\partial B} \sigma_{B}\right)^{2}=\frac{4 A B}{(A+B)^{3}} .
\end{gathered}
$$


The SNR we are optimizing is $C / \sigma_{C}$,

$$
\mathrm{SNR}=\frac{C}{\sigma_{C}}=\frac{(A-B) \sqrt{A+B}}{2 \sqrt{A B}}=\frac{\sqrt{k}}{2} \frac{(a-b) \sqrt{a+b}}{2 \sqrt{a b}} .
$$

We note that the SNR increases with the square-root of the incident photon flux. For a given flux level, we can optimize the design based on Eq. 5 .

\section{RESULTS}

In the aligned state the open regions of the FS grating are centered on the bright regions of the aerial image for maximum transmission. To account for the FS thickness $(80-100 \mathrm{~nm})$, transmission efficiencies are calculated through $z$, and the (longitudinal) position of peak transmission is identified for each set of design parameters. Within the plane where the maximum transmission occurs, the minimum transmission is calculated from the anti-aligned state. Together, these pairs of transmission values are be used to calculate the measurement contrast of the FS.

\subsection{Transmission and contrast.}

Figures 3 and 4 show the maximum and minimum transmission values for various FS open widths and thicknesses. The transmitted flux values are normalized to the input flux level: these fractional-transmission values thus represent the efficiency of the ideal system, with an unaberrated lens and a perfect, binary mask. The data show that the most significant design parameter is the open-width of the FS grating. Below 32-nm open-width the transmission decreases more quickly due to the high aspect ratio and absorption in the walls of the thick grating. Thicker gratings also decrease transmission, but the effect is not strong in the 80-100-nm thickness range of interest.

It is interesting to compare the results of the detailed calculation with those from an simple model based on a thin opaque screen as the FS grating. In Figs. 3, 4 and 6, the dashed lines show transmission values in the simple model. The thinscreen model significantly over-predicts the minimum transmission indicating that the thick FS blocks most of the transmitted light in the anti-aligned state. Furthermore, the thin-screen model overestimates the transmission at small openwidths; again, this is due to thickness effects. Perhaps unexpectedly, the thick-FS model predicts higher transmission efficiency than the thin screen model for open-widths above approximately 28 -nm. This can be explained by the incomplete absorption of light at the interior edges of the FS (compared with the perfectly opaque thin screen model); diffracted light near the boundaries can reach the open area and be transmitted through.

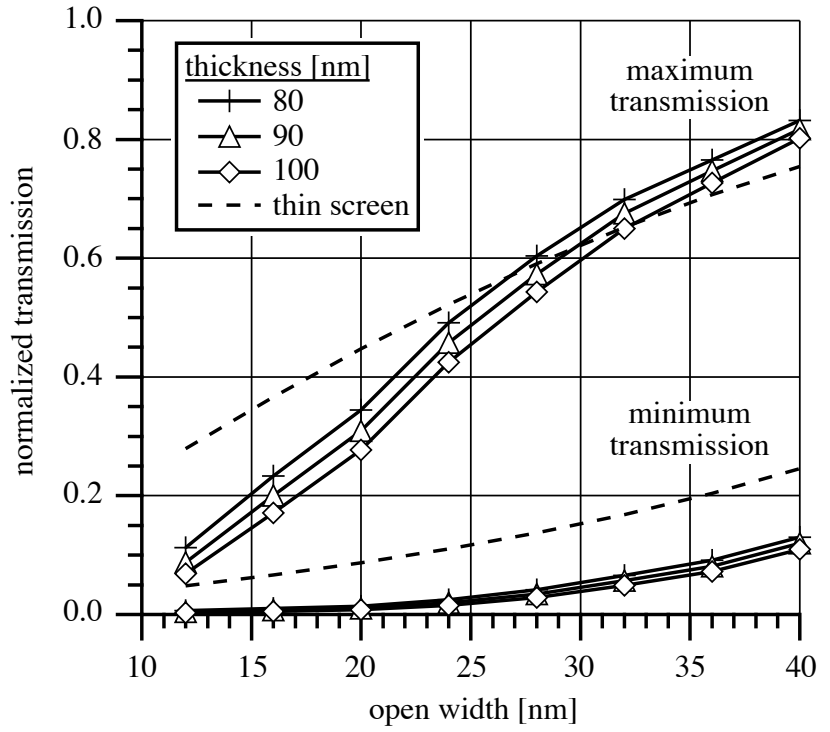

Fig. 3. Maximum and minimum FS transmission values normalized to the input flux.

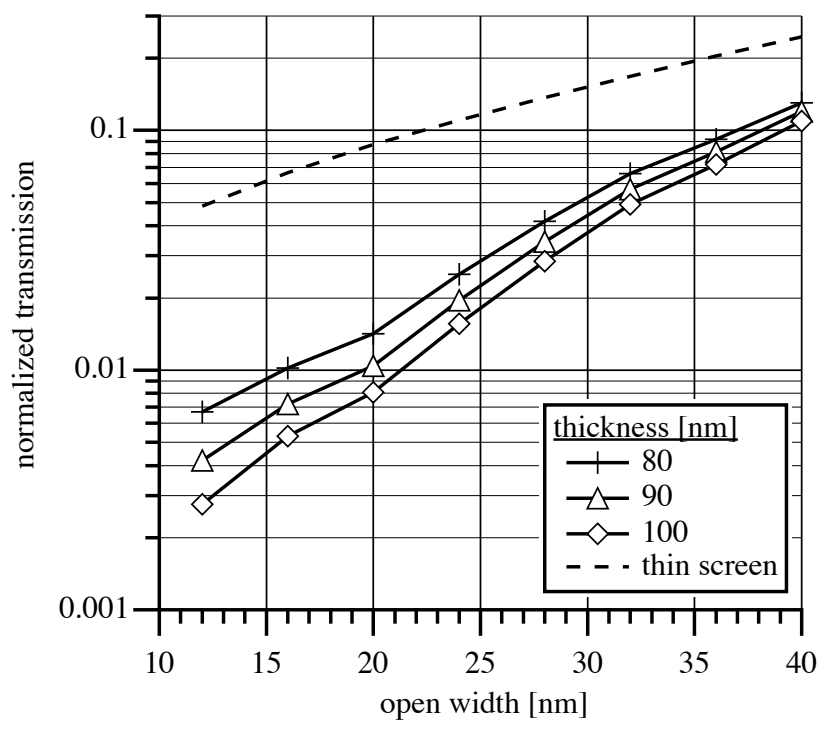

Fig. 4. Minimum FS transmission values from Fig. 3, shown on $\log$ scale. 
With very low transmission in the anti-aligned state, the measurement contrast of the FS can be higher than that of the inherent field contrast being sampled. Figure 5. shows the predicted measurement contrast through $z$ for 80 -nm-thick FS open width and different open-width values from 12 to $40 \mathrm{~nm}$. The narrower open-width designs yield slightly higher measurement contrast than the larger open-widths, but as Fig. 3 shows, this comes at the significant expense of flux.

Owing to the thickness of the FS, there is a small longitudinal offset between the plane that includes the top (entrance) side of the FS and the image plane, where the inherent field contrast is maximized: the FS finds maximum measured contrast when the image plane occurs within the thickness of the grating. Figure 5 shows that this longitudinal offset is between $21.2-28.7 \mathrm{~nm}$ in all cases.

Figure 6 shows the peak (through $z$ ) measured contrast for each set of design parameters. The predictions of the simple thin-screen model are shown as a dashed line. Owing to the increased light absorption in the anti-aligned state, the measured contrast in the thick-FS model is higher than the simple model predicts. Where the simple model predicts a monotonic dependence on the open-width, the thick-FS model predicts that the peak contrast is at approximately 20-nm openwidth for all thicknesses studied..

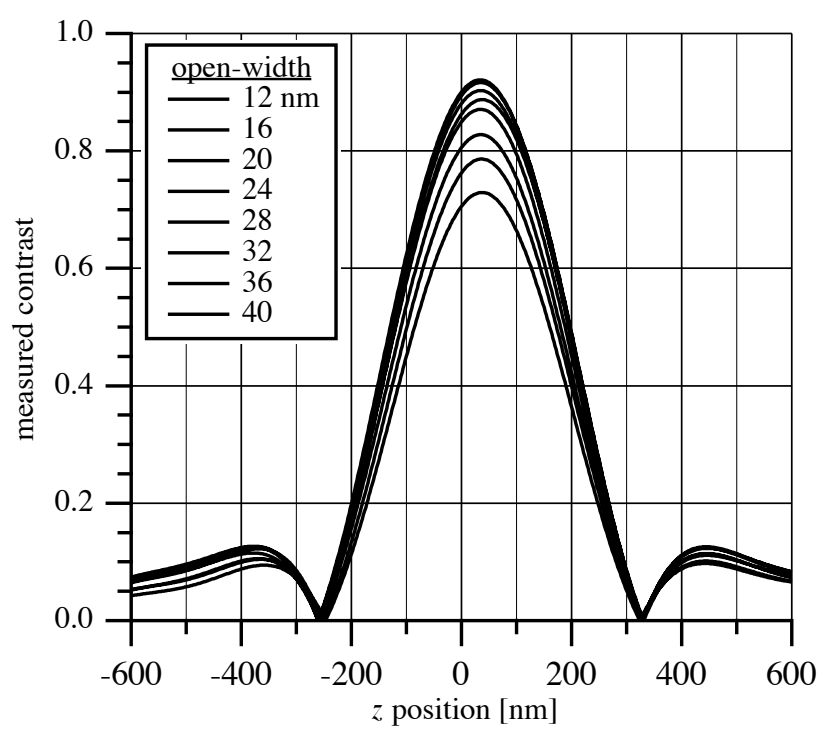

Fig. 5. Predictions of the measured contrast through $z$ for the 80nm-thick FS. The $z$ position is defined with respect to the top surface of the FS. The order of the curves in the legend from top to bottom matches the order of the curves at their peaks.

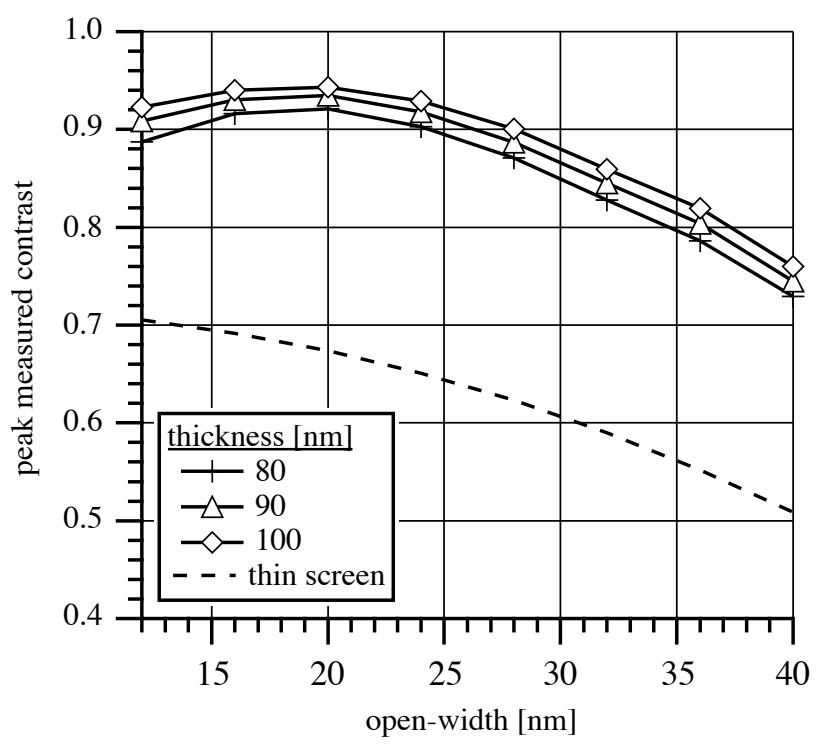

Fig. 6. Predicted peak measured contrast for a variety of FS design parameters. For each thickness, the maximum contrast occurs close to 20-nm open-width. Predictions of the thin-screen model are also shown.

\subsection{Optimized Design.}

Based on our model, the optimized design parameters can be determined using the calculated transmission values and the measurement-contrast optimization formulation described in Section 3. Figure 7. shows the calculated SNR values scaled by the input flux level, $k$. (Since higher input flux levels uniformly improve the SNR, the flux is scaled out of the calculation.) For the three FS thicknesses under consideration, the peak SNR occurs close to 24-nm open-width. The 100nm-thickness design has the highest SNR. Away from the peak, the SNR values decrease by only a few percent when the open-width changes by $\pm 4 \mathrm{~nm}$. Likewise, the variation with the FS thickness is similarly small. In practice, higher SNR can be achieved by increasing photon flux (i.e. measurement time, source intensity, or both).

\section{ANGULAR SENSITIVITY}

Proper rotational alignment between the projected grating pattern and the FS is essential for operation with optimal flux throughput. Here, we estimate the angular sensitivity of the FS. As fabricated, the length of the FS grating lines will be $100 \mu \mathrm{m}$ total. It is anticipated that the demagnified mask pattern will be longer than the FS to make alignment easier. For 
an order of magnitude estimate, we note that a relative azimuthal rotation of $1.6 \mathrm{mrad}$ shifts the projected pattern by one FS grating cycle at the edge of the grating: the allowable rotation tolerance should be a fraction of this angle.

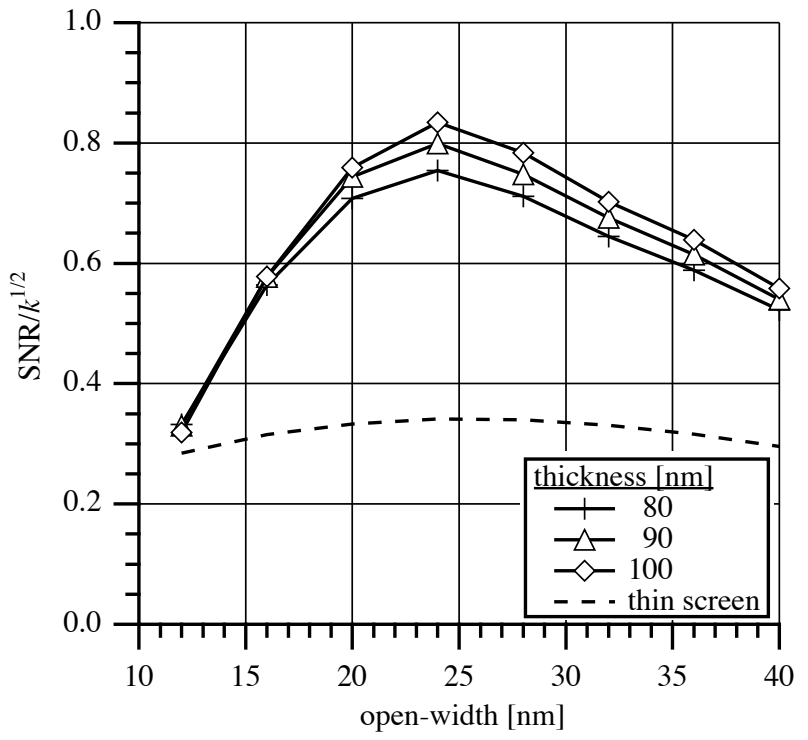

Fig. 7. Predicted signal-to-noise ratio in the contrast measurement for a variety of FS design parameters. Values are scaled to be independent of the photon flux, $k$. The optimal FS designs occur at the peaks.

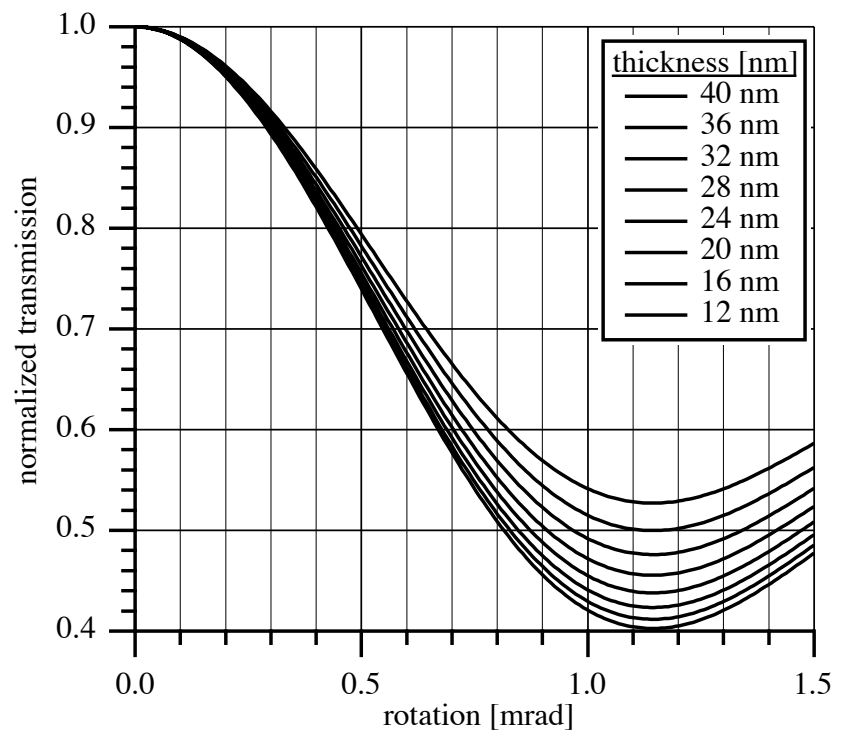

Fig. 8. Estimated azimuthal angular sensitivity of FS with various open-width values.

With arbitrary azimuthal orientation, a sinusoidal intensity pattern is projected onto the FS, modeled as a thin opaque screen grating with various open-width values. The lateral alignment is fixed at the center of the FS. Figure 8. shows the intensity transmission through the FS for eight different grating open-widths; each trace is normalized to its own maximum value. Each FS shows the same nominal behavior: in order to maintain greater than $90 \%$ of peak throughput, the rotational alignment must be within approximately $0.3 \mathrm{mrad}\left(0.017^{\circ}\right)$; similarly $0.45 \mathrm{mrad}\left(0.026^{\circ}\right)$ alignment is required to maintain $80 \%$ of peak throughput. FS designs with narrower open-width values are slightly more sensitive to misalignment. However, peak throughput is only one measure of the angular sensitivity. In further analysis, beyond the scope of this paper, we could also consider the effect of angular misalignment on the measured contrast.

\section{CONCLUSION}

We have presented the design optimization of an EUV focus sensor (FS) consisting of a simple, linear transmission grating translated in the image plane of an EUV projection optic. A mask pattern of lines and spaces forms a partially coherent aerial image with a spatial frequency that matches the FS. To identify the image plane position, the FS is translated laterally and longitudinally within the projected field as the transmitted flux is recorded. The measured contrast is distinct from, and can be higher than, the inherent field contrast due to thickness effects and increased absorption in the anti-aligned state. Optimization of the FSdesign was performed by maximizing the signal-to-noise ratio in the contrast measurement within the design parameter space of interest.

Although only a single case was presented here, the FS performance will depend sensitively on the numerical aperture and pupil shape of the projection lens, and on the mask illumination conditions (i.e. source angular profile). The design parameter space was constrained to a 0.3-NA, annular pupil (matching that of the Micro-Exposure Tools now in use in several laboratories) and annular, $\sigma=0.5-0.7$, illumination.

The FS performance analysis presented here shows that an optimized tool can be created using an 80-nm pitch grating with created in a 100-nm-thick Ni absorber with 24-nm open-width lines (30\% open). While such structures are challenging to fabricate to exact specifications, our predictions show that the device performance is relatively insensitive to small deviations in absorber thickness or open-width from the optimal design parameters. Narrow perpendicular "sup- 
port" features may be included in the design to hold the narrow grating lines apart; these should have only minimal effects on the device performance.

Future analysis should include different FS pitch values, and could include line-edge roughness in the as-fabricated tools. Analysis of the FS performance in a lithographic system with realistic wavefront aberration types and magnitudes would improve the assessment of real-world behavior and establish whether such a tool could be used for aberration characterization.

\section{ACKNOWLEDGMENTS}

The authors gratefully acknowledge Eric Gullikson of CXRO for insightful discussions and assistance with experimental studies, now in progress; and Patrick Naulleau for advice on modeling the partially-coherent illumination. We are also grateful to Bryan Rice of Intel Corporation who funded and guided this research.

\section{REFERENCES}

1. P. Naulleau, K. A. Goldberg, J. P. Cain, E. Anderson, P. Denham, K. Jackson, S. Rekawa, F. Salmassi, G. Zhang, "EUV Microexposures at the ALS Using the 0.3-NA MET Optic," J. Vac. Sci. and Technol. B 22 (6), 2962-65 (2004).

2. H. Hopkins, "The concept of partial coherence in optics," Proc. of the Royal Society of London. Series A, Mathematical and Physical Sciences 208 (1093), 263-77 (1951).

3. H. Hopkins, "On the diffraction theory of Optical Images," Proc. of the Royal Society of London. Series A, Mathematical and Physical Sciences 217 (1130), 408-32 (1953).

4. A. K. Wong and A. R. Neureuther, IEEE Trans. Semicond. Manuf. 8, 419 (1995).

5. T. Pistor, "Electromagnetic Simulation and Modeling with Applications in Lithography," doctoral dissertation, EECS Department, University of California, Berkeley, 2001.

6. J.-P. Berenger, "A Perfectly Matched Layer for the Absorption of Electromagnetic Waves", Journal of Computational Physics, 114 , p. 185-200 (1994). 